\title{
Modelling of Guided Wave Propagation with Spectral Element: Application in Structural Engineering
}

\author{
Ying WANG ${ }^{1, a}$ and Hong $\mathrm{HAO}^{2, \mathrm{~b}}$ \\ ${ }^{1}$ School of Engineering, Deakin University, 75 Pigdons Rd, Waurn Ponds, VIC 3216, Australia \\ ${ }^{2}$ School of Civil and Recourse Engineering, the University of Western Australia, 35 Stirling Hwy, \\ Crawley, WA 6009, Australia \\ a ying.wang@deakin.edu.au , b hong.hao@uwa.edu.au
}

Keywords: Guided wave, Structural Health Monitoring, Spectral element method

\begin{abstract}
Among many structural health monitoring (SHM) methods, guided wave (GW) based method has been found as an effective and efficient way to detect incipient damages. In comparison with other widely used SHM methods, it can propagate in a relatively long range and be sensitive to small damages. Proper use of this technique requires good knowledge of the effects of damage on the wave characteristics. This needs accurate and computationally efficient modeling of guide wave propagation in structures. A number of different numerical computational techniques have been developed for the analysis of wave propagation in a structure. Among them, Spectral Element Method (SEM) has been proposed as an efficient simulation technique. This paper will focus on the application of GW method and SEM in structural health monitoring. The GW experiments on several typical structures will be introduced first. Then, the modeling techniques by using SEM are discussed.
\end{abstract}

\section{Introduction}

During the service lives of civil infrastructures, damage will inevitably occur, such as cracks, corrosion, fatigue, material deterioration, etc. They not only may cause high maintenance investment, but also lead to catastrophic disasters, e.g. structural failures listed in Wikipedia [1]. Therefore, the health condition of a structure should be monitored and maintained. The traditional structural health monitoring (SHM) methods, known as vibration-based methods, are insensitive to minor damage, while conventional non-destructive evaluation (NDE) methods, such as acoustic emission, eddy-current, and ultrasonic techniques, can only cover a small area and thus are not suitable for large civil structures.

Recently, Guided wave (GW) based method has been found as an effective and efficient way to detect incipient damages [2]. GW is a stress wave propagating along a path defined by the material boundaries of a structure. When there are damages in the path, part of the propagating wave will be reflected and part transmitted, resulting in the changes of the received waves. Because of the short wavelength of the generated GW, it is very sensitive to small changes in the structure. Further, GW can propagate a relatively long distance with little attenuation, thus providing a sensing range between those of the conventional NDE techniques and the global vibration-based SHM methods. GW-based methods have been applied to detect damage such as cracks in reinforced concrete (RC) structures [3], additional mass in a rod [4], and debonding between steel bars and concrete in RC structures [5].

Although direct analysis of the recorded GW can lead to damage detection, it is usually difficult to locate and quantify the damage. Basically, it needs accurate and computationally efficient modeling for wave propagation in the structures, to study the effects of damage on the wave characteristics. Different numerical computational techniques have been developed, such as finite element method (FEM) [6] and spectral element method (SEM) [7]. FEM may provide accurate dynamic characteristics of a structure if the wavelength is large as compared to the mesh size. However, the solutions become increasingly inaccurate and computationally expensive as the wave frequency increases, which are usually required for detecting small damages in a structure. This problem can be solved by SEM, where the governing partial differential equations (PDEs) are transferred to ordinary 
differential equations (ODEs) in spatial dimension by using FFT. These ODEs are then solved exactly, and used as the interpolation functions for the spectral element formulation. Therefore, the required number of spectral elements is much less in comparison to the conventional FEM.

In order to explore the applicability of GW based method and SEM in structural engineering, a research group in the University of Western Australia (UWA) initiated a comprehensive project since 2007. This paper will summarize the research achievements till 2012 [8-15], which demonstrate that GW based method is a promising SHM solution and that SEM is an efficient simulation tool.

\section{Methodology}

A typical pitch-catch GW method is used in UWA. As shown in Fig. 1, a pulse signal is sent by a transmitter across the specimen under interrogation and a sensor at the other end of the specimen receives the signal. From various characteristics of the received signal, such as delay in time of transit, amplitude, frequency content, etc., information about the damage can be obtained. In order to perform GW based damage identification, both experimental and numerical studies were carried out.

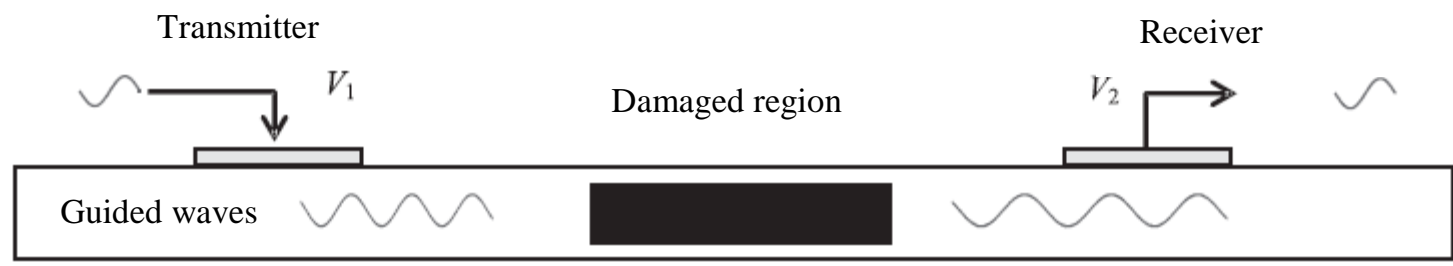

Figure 1: Pitch-catch method

GW test system. A GW experimental system was developed in UWA [8-15], which includes three parts: (i) the computer system together with two data acquisition boards for data transmission and recording; (ii) the actuating module to provide the input of the system, which includes the actuator made of piezoelectric strips and the power amplifier that provides the power supply of the actuator; and (iii) the sensing module to measure the response, which includes the piezoelectric film element and its charge amplifier. The detailed test system can be found in reference [10].

Spectral element method. SEM has been studied over a decade since 1997 [7], and the research has been focusing primarily on mechanical materials, such as composite plate, steel rod, and so on. In order to apply this method to civil infrastructure, intensive research works were conducted in UWA $[8,9,11,12,14]$. Specifically, the concrete-steel interface spectral element (SE), the SE with boundary and discontinuity reflections and the pipe SE were developed. The detailed formulation of those elements can be found from the abovementioned references.

\section{Application in structural damage identification}

In this section, the research works in UWA related to GW and SEM are presented. Extensive experimental and numerical studies were performed by using different structural members.

Steel bar. First, the GW test and SEM simulation were performed on steel rebar. As can be seen from Figure 2(a) and (b), the wave propagation results for different steel bars are almost identical, demonstrating that the developed GW test system is very stable. It is worth noting that due to boundary reflection, there are three received waves by the excitation. This phenomenon is explained in details in reference [9]. The numerical model considering both boundary and discontinuity (damage) reflections was developed and used to simulate the wave propagation. As shown in Figure 2(c), the experimental and numerical results are almost identical. This further demonstrates the effectiveness of SEM simulation.

Steel beam. The techniques were then applied to damage detection of a steel beam of $1.500 \mathrm{~m}$ long and $25 * 25 \mathrm{~mm}$ cross section. Crack damage is simulated by cutting the beam, as shown in Figure 3 (a). The crack width is $1 \mathrm{~mm}$ and its depths are $3.0 \mathrm{~mm}, 6.0 \mathrm{~mm}, 9.0 \mathrm{~mm}$ and $12.5 \mathrm{~mm}$, representing 
different levels of damage. As shown in Figure 3(b), the damage can be clearly detected from GW test results, indicated by waves $g$ and $h$ defined in [9] and [13]. Based on Figure 3(c) and (d), SEM accurately predicted the main wave propagation results under both intact and damaged conditions.
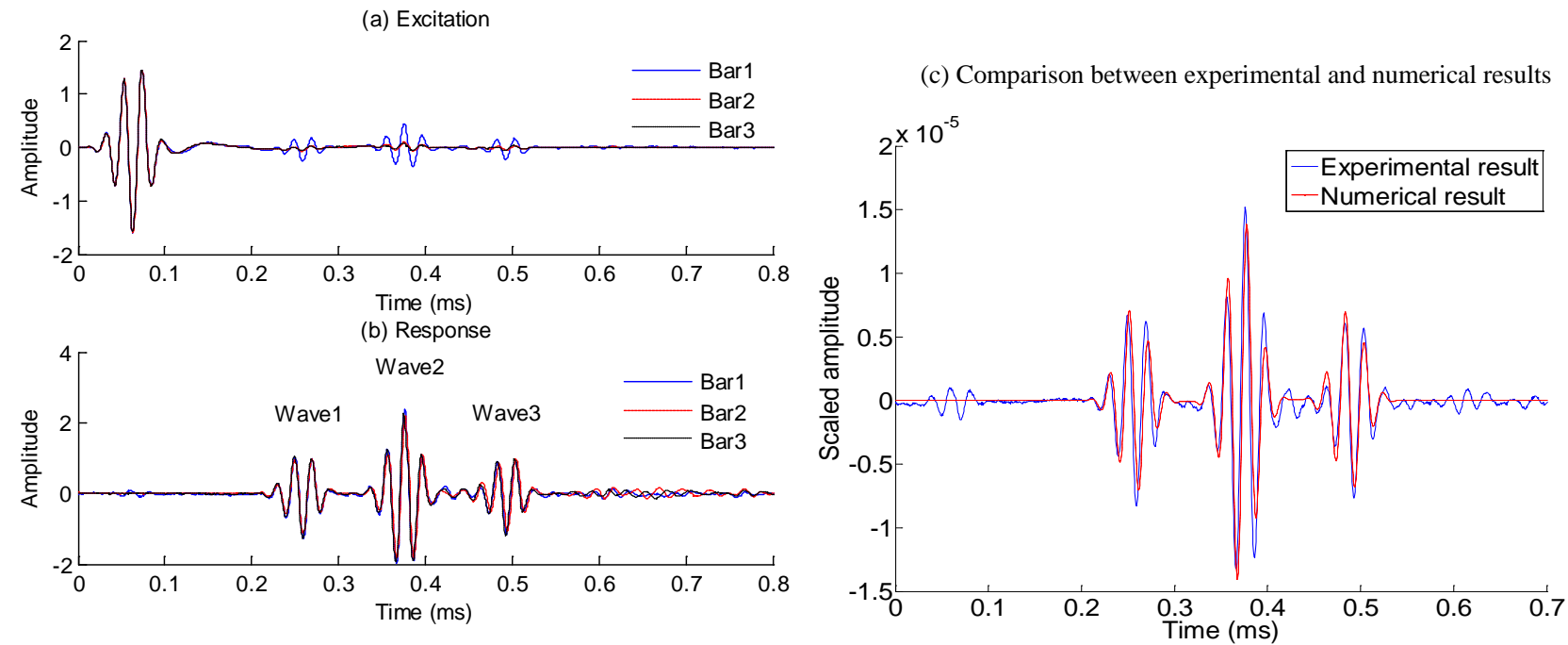

Figure 2: Guided wave propagation results on steel rebar

(a) Experimental configuration

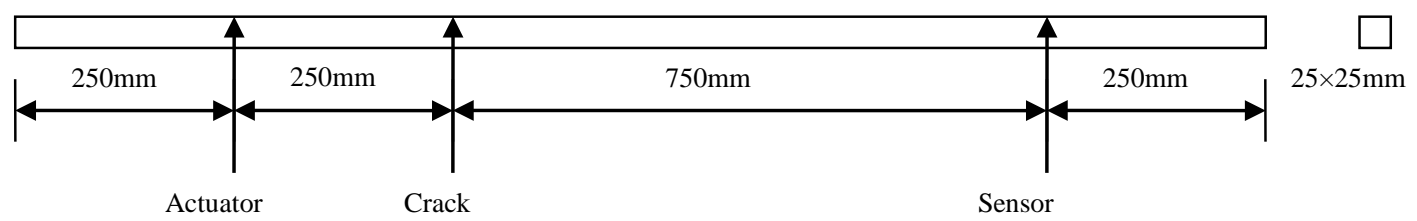

(c) Comparison under intact condition
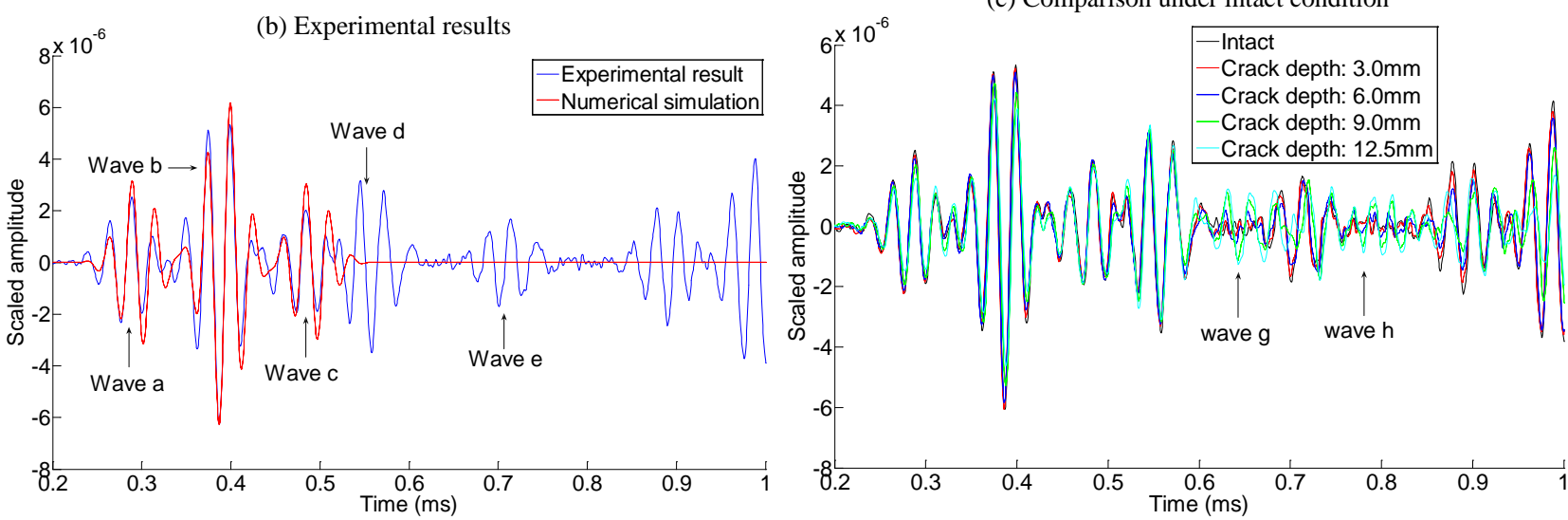

(d) Comparison under damaged condition

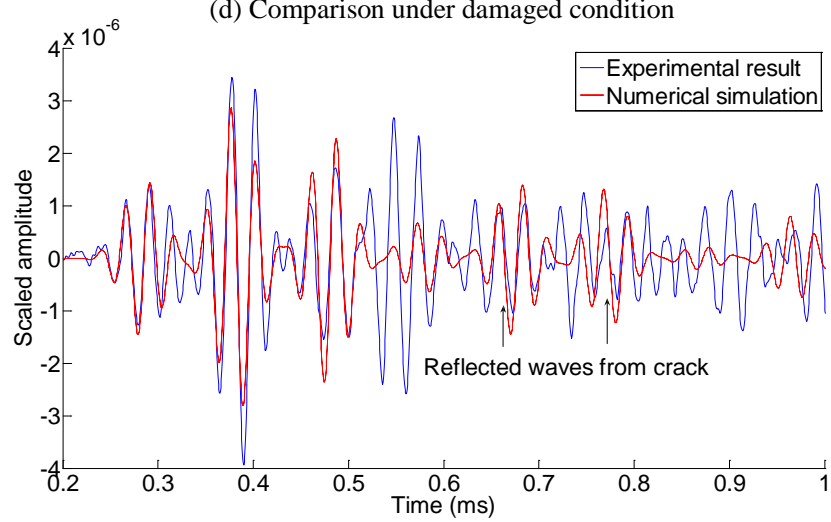

Figure 3: Guided wave propagation results on steel beam 
Concrete-steel interface. In order to detect damages on concrete-steel interface, such as de-bonding, the actuator and sensor were mounted on steel reinforcement bars before casting the RC slabs prepared for experimental tests. The GW test results can be seen in Figure 4(a), (b) and (c). As shown, the GW results are not as stable as those on bare steel rebar. The arrival times of the first few waves show consistency, but the amplitudes seem fluctuant. Compared with bare steel bar, the arrival time of GW measured on reinforcement bar buried inside concrete is much longer, and amplitude much smaller, demonstrating that the wave speed of the concrete-steel interface is much lower, and concrete-steel interface has a much higher damping that leads to faster GW attenuation. .

In order to quantify the changes, a concrete-steel interface SE was developed in UWA. Based on the force analysis on the interface, the equivalent elastic modulus and density of the interface were derived. Numerical simulations were conducted. As can be seen in Figure 4(d), the first arrival wave and the wave with highest amplitude are accurately simulated. This demonstrates that the proposed method is capable of simulating wave propagation results of concrete-steel interface.
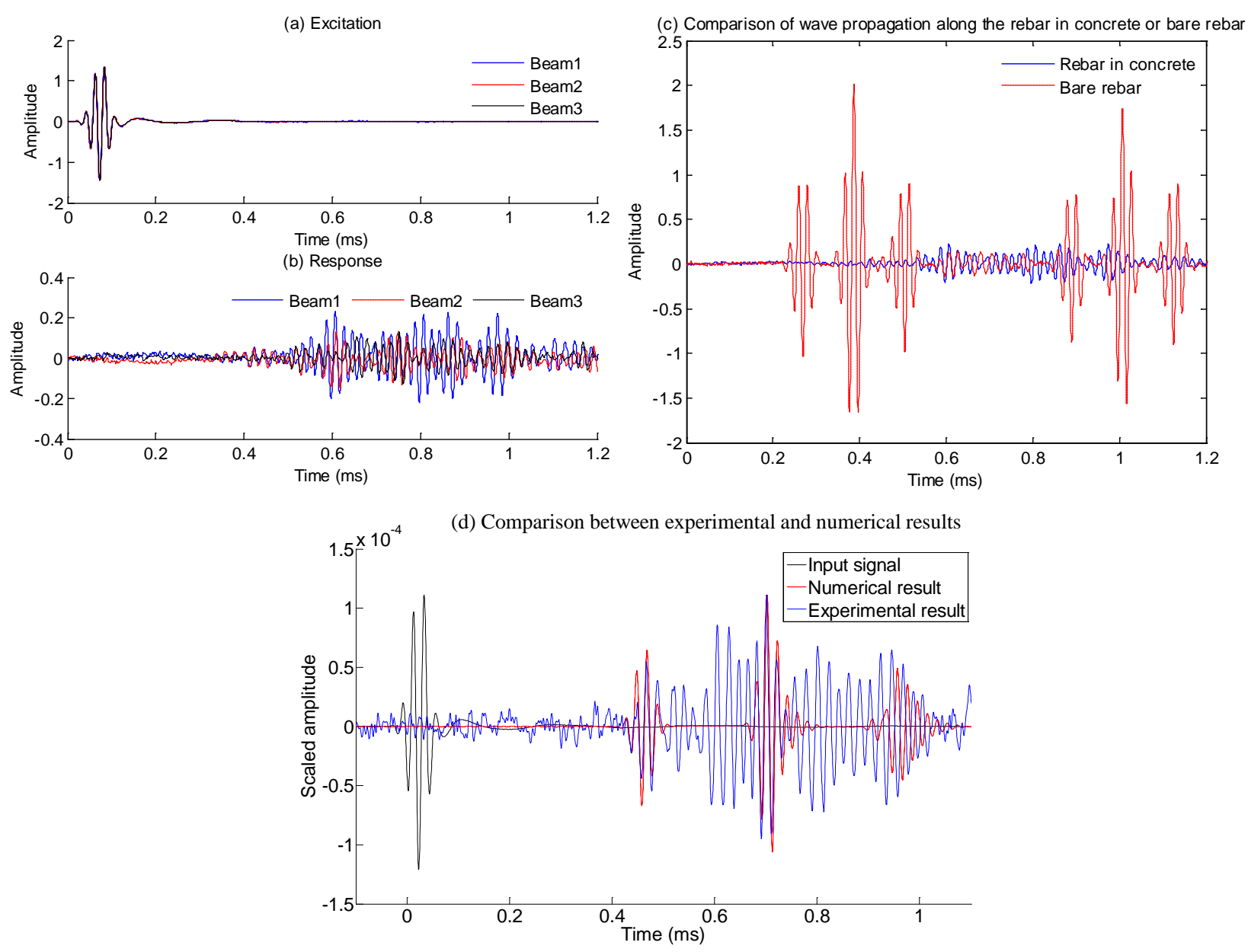

Figure 4: Guided wave propagation results on concrete-steel interface

Further experimental investigation was also conducted on a concrete slab with steel bars with different de-bonding lengths (defined as b), shown in Figure 5. Wavelet techniques were employed to identify the damage location and severity [15].

Steel pipe. The experimental and numerical studies were also conducted on a $3 \mathrm{~m}$ long steel pipe. Due to its circular shape, four sensors are evenly distributed along the circumference at the cross section that is $500 \mathrm{~mm}$ from the end. As can be seen from Figure 6(a) and (b), the wave propagation results from four sensors are very similar, while the late arrival waves show the difference, due to the difference of energy distribution. The influence of cut damage on wave propagation in steel pipe is also studied. When there is cut damage on the pipe $(20 \mathrm{~mm}$ wide vertical cut and located $1000 \mathrm{~mm}$ 
from the right end), the amplitude of the first few waves become lower as shown in Figure 6(c), while an additional wave appears, which can be easily used as a damage indicator.

(a) Experimental configuration

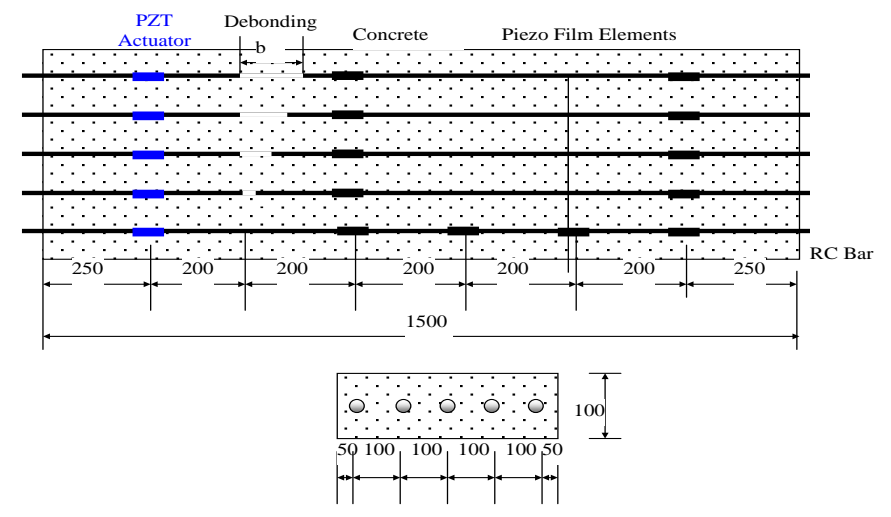

(b) Measurements at $400 \mathrm{~mm}$ from actuator

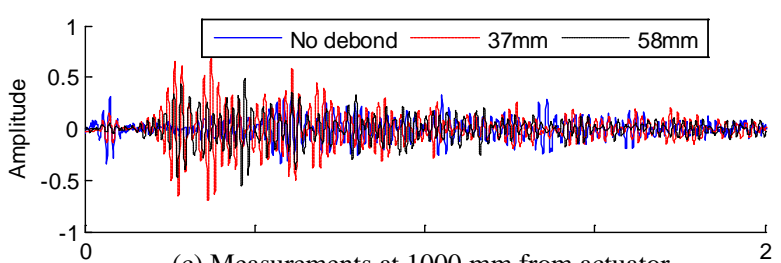

(c) Measurements at $1000 \mathrm{~mm}$ from actuator

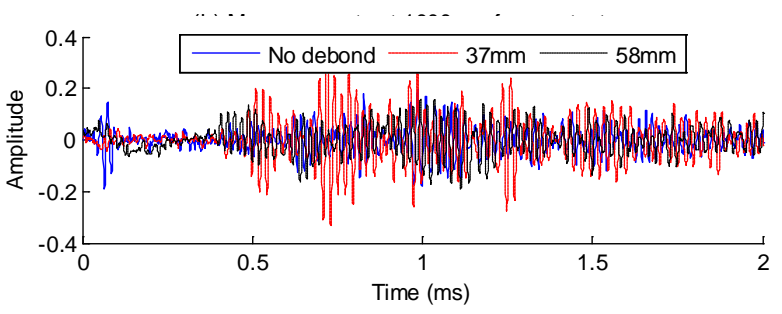

Figure 5: Guided wave propagation tests and results on concrete slab with various debonding lengths
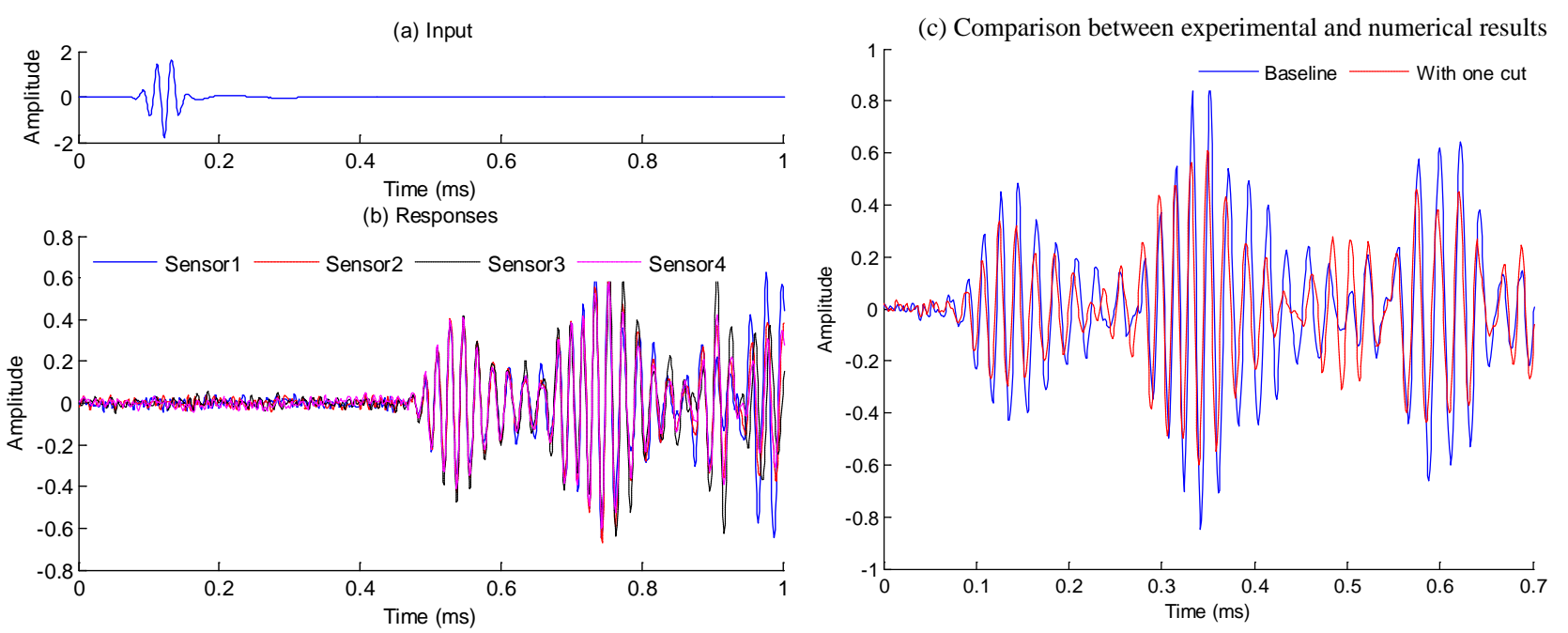

Figure 6: Guided wave propagation results on steel pipe

Discussions. Based on existing results, GW method shows great potential as damage detection approach. It is sensitive to small damages and can cover a relatively long distance. Further, GW method can be used as an active SHM method, since it can interrogate the structure to detect the presence of damage, and to estimate its extent and intensity. Therefore, its application on civil structures should be further investigated.

To simulate wave propagation, SEM is demonstrated to be an effective tool. The development of concrete-steel SE, SE with boundary and discontinuity reflections, and other SEs, enables application of this technique to real SHM cases.

\section{Conclusions}

This paper summarizes the research contributions on GW and SEM in the UWA. Due to the page limit, the research works are only briefly shown. The detailed results and discussions can be found in related publications. Based on the existing results, it can be concluded that GW method is a promising 
technique for structural damage detection and that SEM is a suitable modeling technique for GW. Further investigation on this area should be highly recommended.

\section{References}

[1] Wikipedia, List of Structural Failures and Collapses, http://en.wikipedia.org/wiki/List_of_structural_failures_and_collapses

[2] Raghavan A. and Cesnik C.E.S. Review of Guided-wave structural health monitoring. The Shock and Vibration Digest, 39(2), (2007), 91-114.

[3] Song, G., Gu, H., Mo, Y.L., Hsu, T.T.C. and Dhonde, H. Concrete structural health monitoring using embedded piezoceramic transducers, Smart Materials and Structures, 16(4), (2007), 959-968.

[4] Palacz M., Krawczuk M. and Ostachowicz W. Detection of additional mass in rods: experimental and numerical investigation. Archive of Applied Mechanics, 74, (2005), 820-826.

[5] Wu F. and Chang F.K. Debond detection using embedded piezoelectric elements in reinforced concrete structures-part II: analysis and algorithm. Structural Health Monitoring, 5(1), (2006), 17-28.

[6] Moser F., Jacobs L.J. and Qu J. Modeling elastic wave propagation in waveguides with the finite element method. NDT\&E International, 32(4), (1999), 225-234.

[7] Doyle J.F. Wave Propagation in Structures: Spectral Analysis using Fast Discrete Fourier Transforms. Springer-Verlag, New York. (1997)

[8] Wang Y. and Hao H. Structural damage detection using local and global methods. Advances in Structural Engineering, 15(5), (2012), 807-824

[9] Wang Y., Hao H., Zhu X.Q. and Ou J.P. Spectral element modelling of wave propagation with boundary and structural discontinuity reflections. Advances in Structural Engineering, 15(5), (2012), 855-870.

[10]Wang Y. and Hao H. Integrated health monitoring for reinforced concrete beams: an experimental study. Australian Journal of Mechanical Engineering (special issue for ACAM6: 6th Australasian Congress on Applied Mechanics), 8(2), (2011), 207-217

[11]Wang Y., Zhu X.Q., Hao H. and Ou J.P. Spectral element model updating for damage identification using clonal selection algorithm. Advances in Structural Engineering, 14(5), (2011), 837-856.

[12]Wang Y., Zhu X.Q., Hao H. and Ou J.P. Guided wave propagation and spectral element method for debonding damage assessment in RC structures. Journal of Sound and Vibration, 324, (2009), 751-772.

[13]Wang Y., Zhu X.Q., Hao H. and Fan K.Q. Integrated health monitoring for a steel beam: an experimental study. ICSCS'10: 4th International Conference on Steel \& Composite Structures, 21-23 July 2010, Sydney, Australia.

[14]Wang Y., Zhu X.Q., Hao H. and Fan K.Q. Development and testing of guided wave techniques for pipeline integrity monitoring. SUT 2009: First Annual Subsea Technical Conference, 17-19 February 2009, Perth, Australia

[15] Ou G., Wang Y., Hao H. and Zhu X.Q. Identification of De-bonding between Steel Bars and Concrete using Wavelet Techniques: Comparative Study. Australian Journal of Structural Engineering. 14(1), (2013), 43-56 